\title{
Adequacy of Depression Treatment in Spouses of Cancer Survivors: Findings From a Nationally Representative US Survey
}

\author{
Kristin Litzelman, $P h D^{1,2}$, Abiola O. Keller, PA-C, PhD ${ }^{3}$, Amye Tevaarwerk, MD ${ }^{1,2}$, and Lori DuBenske, \\ $P h D^{1,2}$
}

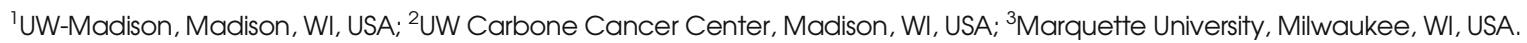

\begin{abstract}
BACKGROUND: Recent research suggests that mental health problems in spouses of cancer survivors are associated with worse mental health in the survivors themselves. Adequately treating spousal mental health problems therefore represents an opportunity to improve outcomes for both cancer survivors and their co-surviving family members.
\end{abstract}

OBJECTIVE: Using nationally representative data, this study sought to determine how depression treatment differs between spouses of cancer survivors with depression compared to the general married population and assess rural/urban disparities in treatment.

DESIGN: The design of the study is cross sectional.

PARTICIPANTS: Data are from the Medical Expenditures Panel Survey, a household-based survey of US adults; we concatenated data from 2004 to 2013. We identified spouses of cancer survivors $(n=225)$ and a comparison group of married adults $(n=3678)$.

MAIN MEASURES: Key measures included depression, guideline concordance of depression treatment (at least four prescriptions related to depression treatment, or at least eight psychotherapy or counseling visits), and sociodemographic characteristics. Logistic regressions evaluated the association between whether their spouse had cancer and receipt of guideline-concordant treatment, controlling for sociodemographic characteristics; secondary analyses included rurality as a moderator. Analyses were weighted to account for the complex sampling design.

KEY RESULTS: Spouses of cancer survivors were 33\% less likely to receive guideline-concordant depression treatment than comparison spouses (odds ratio (OR) 0.67, 95\% confidence interval (CI) 0.45-0.99), controlling for covariates. Rural-urban disparities were observed: rural spouses of cancer survivors were $72 \%$ less likely to receive guideline-concordant treatment (OR 0.28, 95\% CI 0.11-0.68) than rural comparison spouses. Spouses of cancer survivors and comparison spouses were no different in their receipt of any treatment versus no treatment.

CONCLUSIONS: Spouses of cancer survivors with depression may be at increased risk of non-guideline-concordant

Electronic supplementary material The online version of this article (https://doi.org/10.1007/s11606-018-4331-z) contains supplementary material, which is available to authorized users.

Received August 8, 2017

Revised November 16, 2017

Accepted January 4, 2018

Published online February 5, 2018 depression treatment, particularly in rural areas. The findings have implications for identifying and educating individuals with depression in primary care and other clinical areas.

KEY WORDS: caregiver; cancer; co-survivor; survivorship; depression.

J Gen Intern Med 33(6):869-76

DOI: $10.1007 / \mathrm{s} 11606-018-4331-\mathrm{z}$

(c) Society of General Internal Medicine 2018

\section{INTRODUCTION}

There are an estimated 15.5 million cancer survivors in the $\mathrm{USA}^{1}$ (defined as any person diagnosed with cancer, from the time of initial diagnosis ${ }^{2}$ ). Given that the adverse impacts of cancer extend beyond the cancer patient themselves, ${ }^{2,3}$ millions more family members are considered "co-survivors" of the disease. Co-survivors often serve as informal cancer caregivers, playing a critical role in the well-being of individuals with cancer. In addition to supporting the survivor through their illness, co-survivors often struggle with their own emotional distress. The caregivers of cancer survivors have been found to have equal, if not greater, rates of depression than the survivors themselves (12-59\%). ${ }^{4}$ Spouses in particular often experience greater burden, strain, or distress than other family caregivers $^{5,6}$ and are more likely than the general population to experience depression. , $^{8}$

Recent research has shown that when their spouses experienced depressed mood, cancer survivors were four times more likely to become depressed over the next year, ${ }^{9,}{ }^{10}$ supporting theoretical and emerging empirical work around the interrelationship between families/caregivers and cancer survivors. ${ }^{11}$ Given the association between depression in cancer survivors and adverse outcomes ranging from decreased medication adherence to premature mortality, ${ }^{12-17}$ appropriately treating spousal depression may afford an opportunity to improve outcomes for both cancer survivors and their spouses. However, despite the existence of effective pharmacotherapy and psychotherapy treatments, ${ }^{18}$ many cancer caregivers (including spouses) may be at risk for under-treatment of depression, ${ }^{19}$ as informal caregivers often report struggling with selfcare $^{20}$ and likely face time, cost, and access constraints that may be barriers to receiving health care. Furthermore, low socioeconomic status has been associated with less adequate 
depression treatment ${ }^{21}$ and rural populations face known mental health treatment challenges. ${ }^{22}$ Yet, we know very little about the unique needs and outcomes of rural caregivers.

This study sought to evaluate how cancer caregivers with depression currently receive treatment in the USA. Specifically, we sought to determine how treatment patterns for spouses of cancer survivors differ from the general population, and whether there are socioeconomic or geographic disparities in treatment rates. Better understanding treatment patterns and disparities among cancer caregivers will inform medical practice and caregiver education, with important implications for the wellbeing of cancer survivors and their families.

\section{METHODS}

Data were obtained from the Medical Expenditures Panel Survey (MEPS). The MEPS is a household-based, nationally representative survey of US adults. Each year, the MEPS collects data on a new panel of households and each panel is followed for five time points over a 2.5 -year period. For this study, we concatenated data from panels 9-17 collected between 2004 and 2013. Detailed information about MEPS methodology and data is available at http://meps.ahrq.gov.

\section{Sample Selection}

MEPS respondents were eligible for this study if they (1) were married to someone with or without cancer and (2) reported having depression. To identify depressed spouses of cancer survivors, we first used the MEPS medical conditions file to identify adults who had a cancer-related health-problem, medical event, or disability. Adults with any type of cancer or malignancy (excluding those with only non-melanoma skin cancer) were categorized as survivors. All other adults were categorized as general population.

We then linked both survivors and the general population with their spouses (if applicable) using a spousal ID. Depression status was then determined using the Conditions Enumerations section of the MEPS, in which participants reported their physical and mental/emotional conditions, as well as conditions related to any medical events or disabilities. Using this information, MEPS coders assign truncated ICD-9 codes. Participants with ICD-9 code 296 or 311, during any round, were classified as having depression. All depressed spouses of cancer survivors were included in the final sample; couples in which both spouses had cancer were excluded. In the comparison sample of depressed spouses in the general population, when couples both reported depression an index spouse was randomly selected. This resulted in an eligible sample of 4001 adults $(n=229$ spouses of cancer survivors, $n=3772$ comparison spouses; Fig. 1).

\section{Key Measures}

Dependent Variable: Depression Treatment. Based on guidelines from the 2010 American Psychological
Association $^{23}$ and the definitions used in previous research, ${ }^{21}$, ${ }^{24-26}$ guideline-concordant treatment of depression was defined as receiving at least four prescriptions related to depression treatment or at least eight outpatient or office-based psychotherapy or counseling visits. Using the MEPS prescription file, we identified whether a respondent received prescriptions indicated for the treatment of depression as defined by Multum Lexicon Drug Database (subclassification code 249). ${ }^{27}$ MEPS respondents reported the name of any prescribed medicine they purchased or otherwise obtained during each round. Each original purchase and any subsequent refills were recoded as distinct medications. Using the MEPS Outpatient Department Visits and MEPS Office-Based Medical Provider Visits files, we identified the number of visits involving psychotherapy. Prescriptions and psychotherapy visits were counted over a rolling three-round period (i.e., time points $1-3$, time points $2-4$, and/or time points $3-5$ ); four prescriptions or eight psychotherapy visits in any period were counted as receiving guideline-concordant treatment. Prescriptions were assumed to be for a minimum of 30 days, and psychotherapy for a minimum of $30 \mathrm{~min}$. We also categorized respondents who had received any treatment versus no treatment for depression.

Independent Variable: Co-Survivor/Comparison Status. Cosurvivors (here referring specifically to spouses of cancer survivors) and comparisons (spouses in the general population) with depression were identified as described above.

Covariates. Following Keller et al., ${ }^{26}$ predisposing, enabling, and need factors were considered as covariates. ${ }^{28}$ Predisposing factors included age, gender, race/ethnicity (white non-Hispanic versus other), education (high school or less versus some college or more), employment status (employed versus not employed), percent of the federal poverty level $(100,200,300$, or $400 \%+)$, rurality (Metropolitan Statistical Area, rural versus urban), and region of residence (Northeast, Midwest, South, or West). Enabling factors included health insurance coverage (any public, private only, or uninsured) and having a usual source of care (yes versus no). Need factors included number of health conditions, self-reported health (good/fair/poor versus excellent/very good), health service use in the past 12 months (low $<4$ visits versus high 4+ visits), and level of depressed mood (Patient Health Questionnaire- ${ }^{29}$ ). Covariates were measured at baseline (round 1); if baseline data were missing, the variable was back-filled with data from the next available time point. For co-survivors, we also assessed the survivor's cancer type (blood, breast, colorectal, prostate, other, or multiple) and time since diagnosis $(<5$ years, $5+$ years, or unknown/unreported) as reported in the medical conditions file; these data were for descriptive purposes only.

\section{Analytic Approach}

Descriptive statistics (cross-tabulations; means and standard deviations) were calculated on all covariates. Co-survivors and 


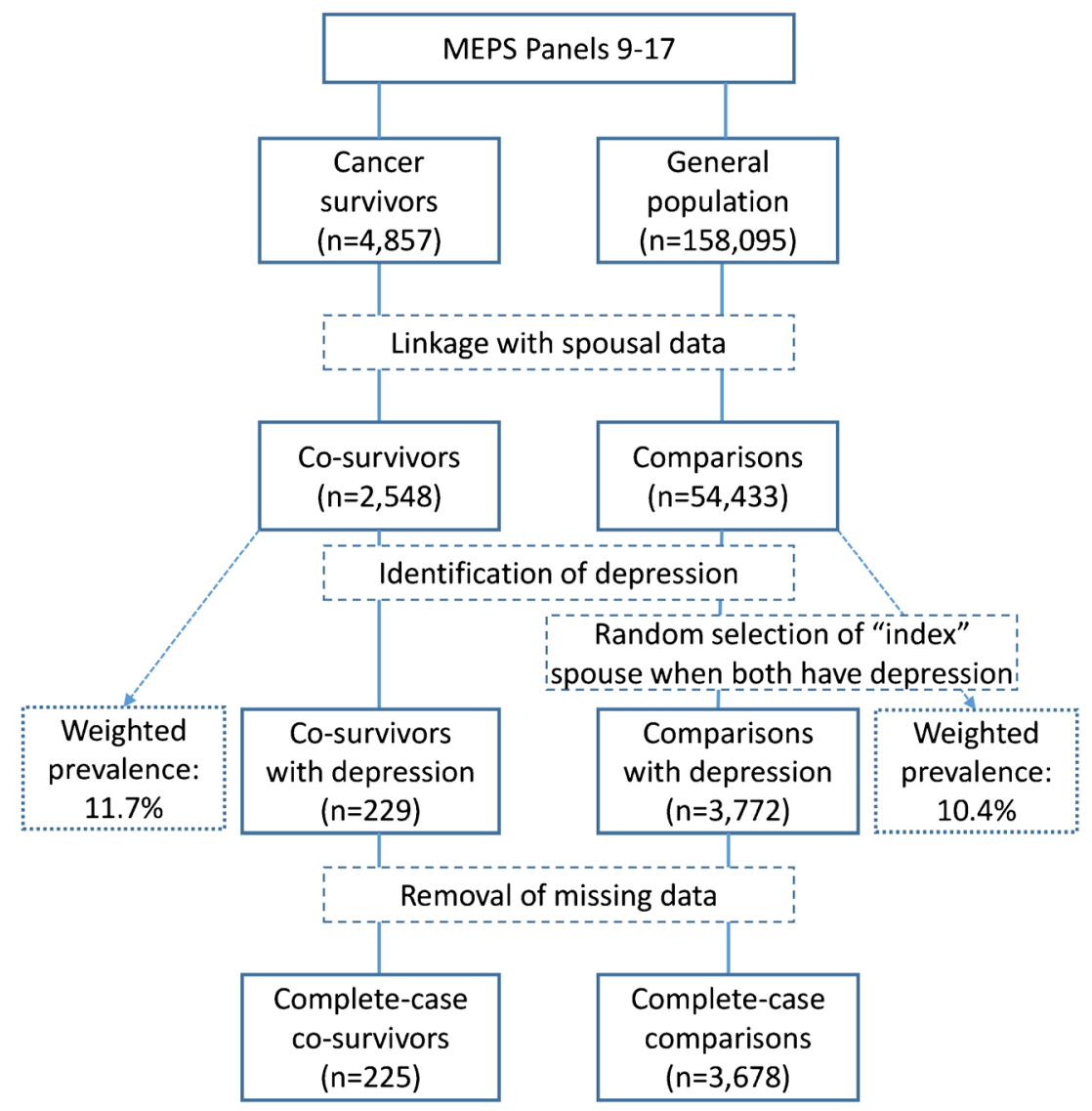

Figure 1 Identification of eligible participants. MEPS Medical Expenditures Panel Survey. Data are from 2004 to 2013.

comparison spouses were compared on all covariates using chisquared and $t$ tests. Among co-survivors and comparison spouses with depression, we calculated the proportion receiving guideline-concordant treatment, and any treatment. We then ran a bivariate logistic regression, regressing receipt of guidelineconcordant treatment on co-survivor/comparison status. The predisposing, enabling, and need covariates and panel number were then added to this model in a multivariable logistic regression. Finally, moderation was assessed by including interaction terms for (1) rurality*co-survivor status and (2) poverty*cosurvivor status in separate regressions. All analyses were conducted in SAS (v. 9.4) using survey weighting procedures to adjust for the complex sampling frame of the MEPS.

Approach to Missing Data. This study was conducted as a complete case analysis, and those with missing covariate data were dropped from the analysis ( $n=4$ spouses of cancer survivors $(1.8 \%)$ and $n=94$ comparison spouses $(2.5 \%)$ ). Those dropped due to missing data were slightly older (53 versus $49, p<0.01)$, more likely to be from the western USA (38 versus $25 \%, p=0.04$ ), less likely to have a usual source of care ( 78 versus $87 \%, p<0.01)$, and had fewer health conditions (7 versus $9, p<0.01)$. They were also less likely to receive guideline-concordant depression treatment (50 versus $62 \%, p=$ $0.01)$. They did not differ significantly on other covariates.
Sensitivity Analysis. To assess whether our findings are robust to our operationalization of guideline concordance, we conducted sensitivity analyses testing six psychotherapy visits (the number often approved by insurance companies) rather than eight.

\section{RESULTS}

Table 1 depicts the characteristics of the samples. Co-survivors were older than comparison spouses (mean ages 61 versus 50 years, $p<0.0001)$. Almost two thirds $(66 \%)$ of the comparisons were female, while $58 \%$ of the co-survivors were female $(p=0.07)$. Comparisons were also more likely to be employed (56 versus $39 \%, p<0.01$ ). Most participants in both groups were white non-Hispanic and reported at least some college education. Approximately $30 \%$ of participants reported incomes at or below $200 \%$ of the federal poverty level, and approximately $80 \%$ lived in an urban area. Co-survivors were more likely to have only private insurance (21 versus $14 \%$ ), while comparisons were more likely to be uninsured ( 8 versus $3 \%, p<0.0001$ ). Nearly all participants $(\sim 90 \%)$ reported having a usual source of care. Co-survivors reported more health conditions than comparisons (mean conditions: 12 versus 9, $p<0.0001$ ). Cosurvivors were also more likely to report poor self-rated health 
Table 1 Survey Weighted Characteristics of Adults With Depression, Medical Expenditures Panel Survey (2004-2013)

\begin{tabular}{|c|c|c|c|}
\hline & $\begin{array}{l}\text { Co- } \\
\text { survivors }\end{array}$ & Comparisons & $p$ value \\
\hline Weighted number & 582,548 & $9,165,501$ & \\
\hline \multirow[t]{2}{*}{ Unweighted number } & 225 & 3,678 & \\
\hline & $\begin{array}{l}\text { Mean (SD) } \\
\text { or \% }\end{array}$ & $\begin{array}{l}\text { Mean (SD) } \\
\text { or } \%\end{array}$ & \\
\hline \multicolumn{4}{|l|}{ Sociodemographics } \\
\hline Age (years; mean, SD) & $60.93(0.98)$ & $49.44(0.27)$ & $\begin{array}{l}< \\
0.0001\end{array}$ \\
\hline \multicolumn{3}{|l|}{ Gender } & 0.0671 \\
\hline Male & 42.34 & 34.12 & \\
\hline Female & 57.66 & 65.88 & \\
\hline \multicolumn{3}{|l|}{ Race/ethnicity } & 0.5420 \\
\hline Other & 34.71 & 36.95 & \\
\hline White (non-Hispanic) & 65.29 & 63.05 & \\
\hline \multicolumn{3}{|l|}{ Education } & 0.3001 \\
\hline High school or less & 48.25 & 44.08 & \\
\hline Some college or more & 51.75 & 55.92 & \\
\hline \multicolumn{3}{|l|}{ Employment status } & $\begin{array}{l}< \\
0.0001\end{array}$ \\
\hline Not employed & 60.68 & 44.38 & \\
\hline Employed & 39.32 & 55.62 & \\
\hline \multicolumn{3}{|l|}{$\begin{array}{l}\text { Percent of federal poverty } \\
\text { level }\end{array}$} & 0.7076 \\
\hline 100 & 5.67 & 6.43 & \\
\hline 200 & 26.71 & 23.07 & \\
\hline 300 & 25.51 & 28.19 & \\
\hline $400+$ & 42.11 & 42.32 & \\
\hline \multicolumn{3}{|l|}{ MSA } & 0.8267 \\
\hline Rural & 18.22 & 18.93 & \\
\hline Urban & 81.78 & 81.07 & \\
\hline \multicolumn{3}{|l|}{ Region } & 0.4201 \\
\hline Northeast & 22.80 & 15.28 & \\
\hline Midwest & 23.30 & 25.26 & \\
\hline South & 33.49 & 37.18 & \\
\hline West & 20.41 & 22.29 & \\
\hline \multicolumn{3}{|l|}{ Insurance coverage } & $\begin{array}{l}< \\
0.0001\end{array}$ \\
\hline Any public & 75.33 & 77.17 & \\
\hline Private only & 21.21 & 14.48 & \\
\hline Uninsured & 3.45 & 8.35 & \\
\hline \multicolumn{3}{|l|}{ Usual source of care } & 0.1898 \\
\hline No & 7.88 & 11.10 & \\
\hline Yes & 92.12 & 88.90 & \\
\hline $\begin{array}{l}\text { Health conditions (mean, } \\
\text { SD) }\end{array}$ & $11.62(0.59)$ & $9.26(0.13)$ & $<0.0001$ \\
\hline Self-reported health & & & 0.0059 \\
\hline Good/fair/poor & 68.99 & 59.08 & \\
\hline Excellent/very good & 31.01 & 40.92 & \\
\hline \multicolumn{3}{|c|}{ Service use (past 12 months) } & 0.0037 \\
\hline Low (<4 visits) & 37.67 & 49.92 & \\
\hline High (4+ visits) & 62.33 & 50.08 & \\
\hline \multicolumn{4}{|l|}{ Cancer characteristics } \\
\hline Cancer type & & NA & NA \\
\hline Blood & 8.43 & & \\
\hline Breast & 20.20 & & \\
\hline Colorectal & 5.01 & & \\
\hline Prostate & 17.69 & & \\
\hline Multiple & 7.15 & & \\
\hline Other & 41.51 & & \\
\hline \multicolumn{2}{|l|}{ Time since diagnosis } & NA & NA \\
\hline$<5$ years & 45.71 & & \\
\hline $5+$ years & 29.47 & & \\
\hline Unknown/unreported & 24.82 & & \\
\hline Treatment status & & NA & NA \\
\hline $\begin{array}{l}\text { Not receiving treatment } \\
\text { during survey period }\end{array}$ & 64.53 & & \\
\hline $\begin{array}{l}\text { Received treatment } \\
\text { during survey period }\end{array}$ & 35.47 & & \\
\hline
\end{tabular}

SD standard deviation
(69 versus 59\%, $p=0.0059$ ) and high service use in the past 12 months (62 versus $50 \%, p=0.0037$ ).

In the unadjusted data, two thirds of both co-survivors with depression and comparisons reported receiving guidelineconcordant treatment (data not tabled). Table 2 shows the results of the weighted multivariable logistic regression of the association between co-survivor status (i.e., spouses of cancer survivors with depression versus comparison spouses with depression) and receipt of guideline-concordant depression treatment. After controlling for covariates, co-survivors were $33 \%$ less likely to receive guideline-concordant depression treatment (odds ratio $[\mathrm{OR}]=0.67,95 \%$ confidence interval $[\mathrm{CI}]=0.45-0.99$ ). Predisposing, enabling, and need factors were also associated with receipt of guideline-concordant treatment (Table 2; written summary available in Online Appendix).

There was a significant interaction between rurality and cosurvivor status $\left(p_{\text {interaction }}=0.02\right.$ ). Rural co-survivors were $72 \%$ less likely to receive guideline-concordant depression treatment (OR 0.28, 95\% CI 0.11-0.68) than rural comparisons. Figure 2 shows the predicted probabilities of receipt of guideline-concordant treatment, controlling for covariates (with age centered at 50): $40 \%$ of rural co-survivors with depression received guideline-concordant treatment, versus $71 \%$ of rural comparison spouses, $66 \%$ of urban comparison spouses, and $61 \%$ of urban co-survivors.

Poverty level did not moderate the association between cosurvivor/comparison status and guideline-concordant treatment $\left(p_{\text {interaction }}=0.36\right.$; data not tabled). There was no association between co-survivor status and receipt of any depression treatment versus no treatment (Table 3). In sensitivity analyses, changing the definition of guideline concordance from eight psychotherapy visits to six visits did not change the results.

\section{DISCUSSION}

Co-survivors serve as an integral part of the care team for cancer patients, but may also suffer from unaddressed emotional needs. We performed an analysis of an existing database and found that spouses of cancer survivors were less likely to receive guideline-concordant depression treatment than comparison spouses. Our findings support previous research indicating that distressed family caregivers of lung cancer patients underuse mental health services. ${ }^{30}$ In our analysis, co-survivors in rural areas were particularly at risk. We did not observe similar disparities in socioeconomic status, and co-survivors and comparisons with depression did not differ in their receipt of any depression treatment (versus no depression treatment). To our knowledge, this is the first study to evaluate rates of guidelineconcordant depression care among caregivers. 
Table 2 Association Between Spousal Caregiving Status and Guideline Concordant Depression Treatment (Medical Expenditures Panel Survey, 2004-2013)

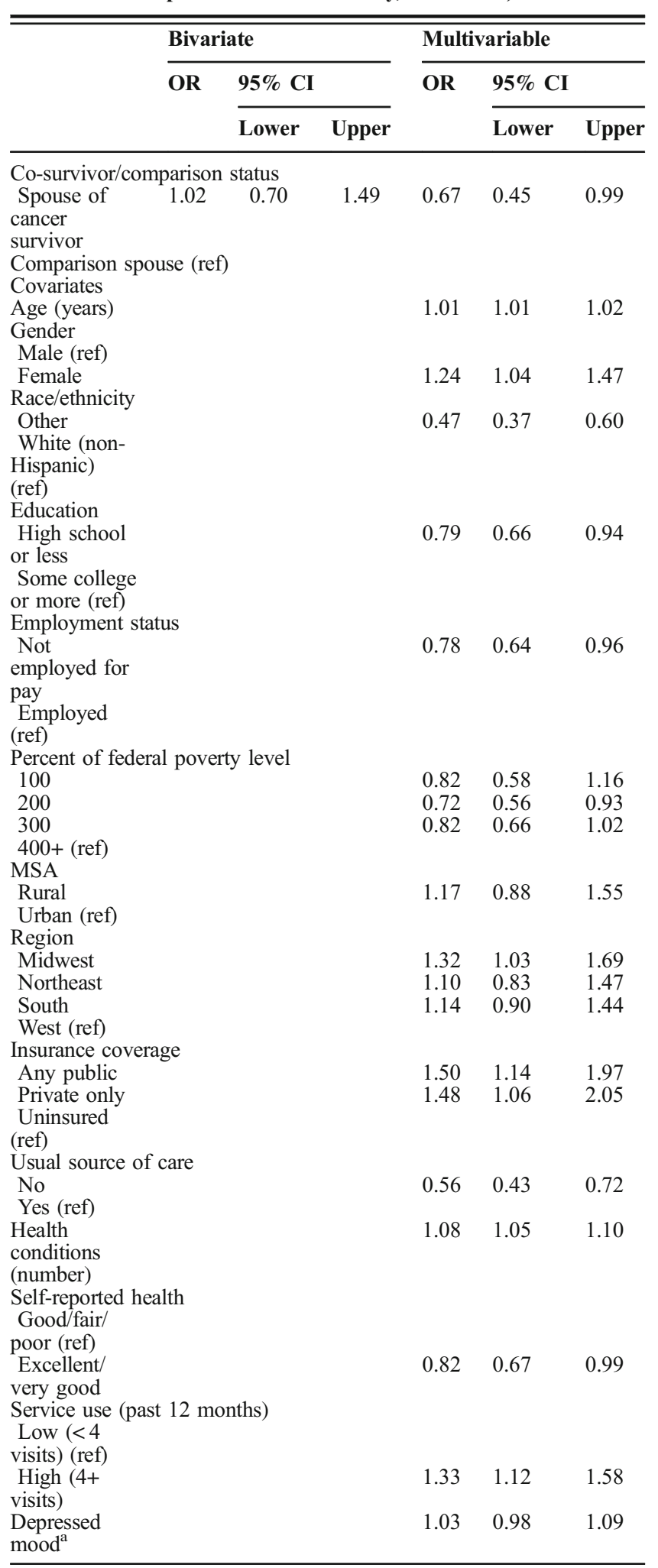

Note: Survey-weighted models; multivariable model also controls for panel number

OR odds ratio, CI confidence interval

${ }^{a}$ Measured by the Patient Health Questionnaire 2 (one-point increase)

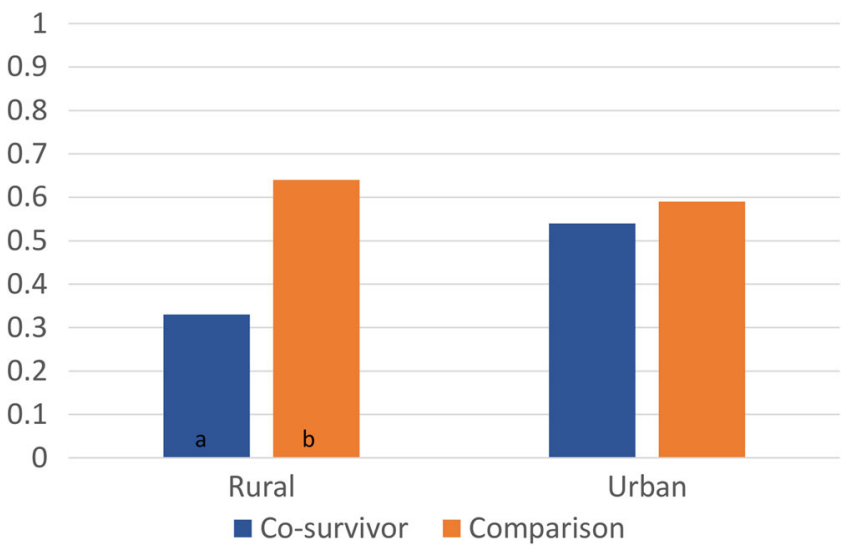

Figure 2 Predicted probabilities of guideline-concordant treatment among spouses of cancer survivors ("co-survivors," blue bars) and comparison spouses in the general population (orange bars), by urbanicity. a Significant difference from rural and urban comparisons $(p<0.05)$; borderline difference from urban co-survivors $(p<$ $0.10)$. b Significant difference from rural co-survivors $(p<0.05)$; borderline difference from urban co-survivors $(p<0.10)$

It is noteworthy that we found differences in guidelineconcordant treatment, even though all eligible subjects reported having depression. One interpretation of this finding is that co-survivors, and particularly rural co-survivors, are at risk for under-treatment of depression. Under-treatment of depression is prevalent among the general population. ${ }^{31}$ Caregivers may be disproportionately affected as they often struggle with self-care ${ }^{20}$ which may extend into mental health care. ${ }^{30}$ Cancer caregivers have reported seeing mental health service use as a "last resort" and prioritizing the care recipient's needs above their own, ${ }^{32}$ potentially leading them to be less likely to seek out or adhere to treatment for their depression.

In addition, expectations and assumptions around cancer and depression may play a role. Anecdotal evidence from our clinical practice suggests that many co-survivors and patients interpret their depression as part of the "cancer experience": they feel that they are supposed to be depressed and assume that it is part of the "necessary suffering" of cancer, and therefore do not seek treatment. They may feel that since they cannot change the circumstances or their survivor's diagnosis, they also cannot change or control their depression. However, to our knowledge, this mindset has not been examined in the literature. Qualitative and quantitative studies are needed to determine to what extent this mindset may act as a modifiable barrier to depression care.

An alternative explanation of these findings relates to differing levels of depression symptoms and/or treatment needs in co-survivors compared to the general population. Although treatment guidelines are a useful tool, they are not meant to supersede clinical judgment. It is therefore possible that lower (guideline non-concordant) levels of treatment were the best course of care for some cosurvivors in this study. Although we controlled for level 
Table 3 Association Between Spousal Caregiving Status and Receipt of Any Depression Treatment (Medical Expenditures Panel Survey, 2004-2013)

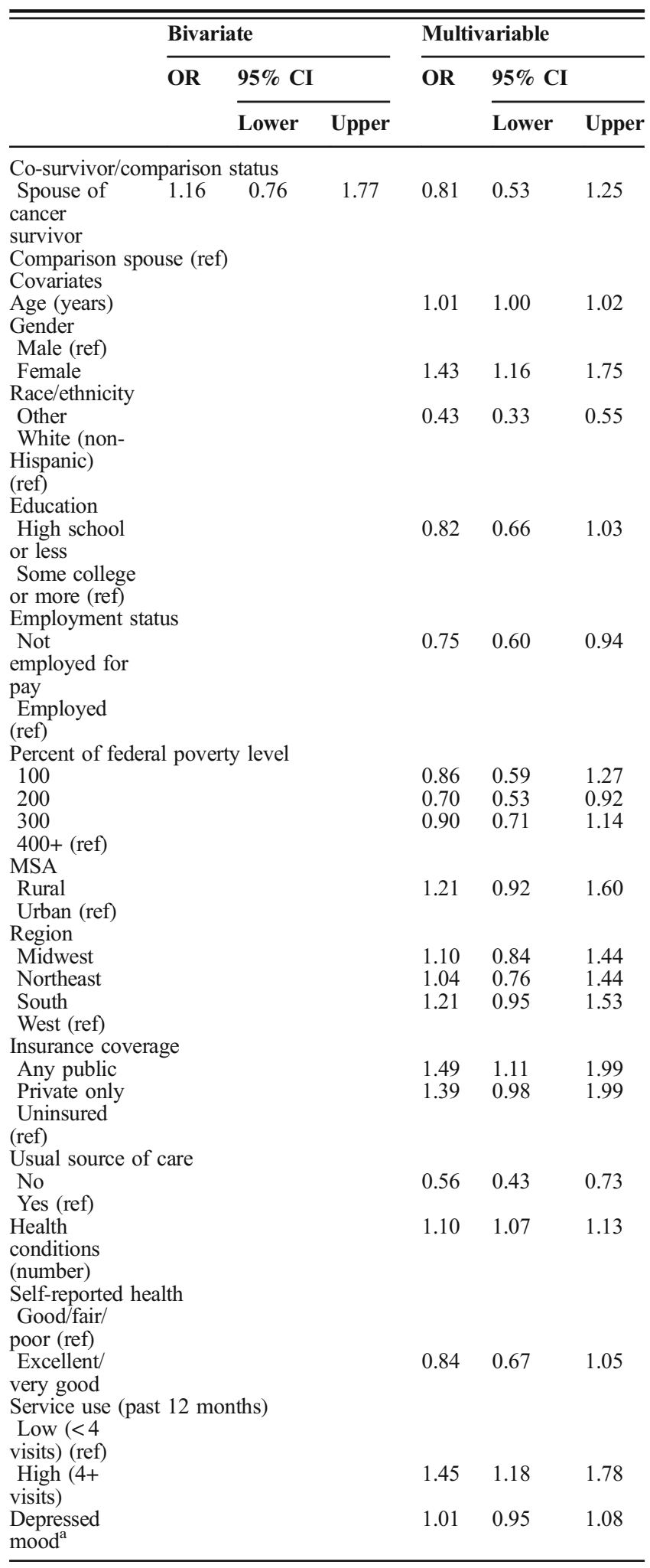

Note: Survey-weighted models; multivariable model also controls for panel number

OR odds ratio, CI confidence interval

${ }^{a}$ Measured by the Patient Health Questionnaire 2 (one-point increase) of depressive symptoms to try to mitigate this confounding factor, studies with richer clinical data will be needed to thoroughly evaluate this possibility.

Rural co-survivors received guideline-concordant treatment at particularly low levels. Previous work in the general population $^{33}$ found no overall rural-urban differences in receipt of depression treatment; similarly, in our study, rural comparisons were statistically no different than urban comparisons to receive treatment. This suggests that caregiving may contribute to under-treatment of depression in rural areas in unique ways. Caregiving may amplify barriers to care observed in rural areas, including the well-documented shortage of mental health providers ${ }^{34,35}$ and possible greater perceptions of stigma and lower belief in the efficacy of and need for treatment in samples from rural versus urban areas. ${ }^{36}$ Caregiving in particular is associated with time and cost burdens; in rural areas, where the geographical distance to a pharmacy or mental health provider may be farther, such time costs may be more likely to present insurmountable barriers to care.

The findings from this study suggest opportunities for improving treatment of depression in cancer co-survivors, ranging from co-survivor education to implications for clinical care. Caregiverspecific interventions, while effective at decreasing burden, have not been successful in reducing caregivers' depression. ${ }^{37}$ This emphasizes the need for guideline-concordant depression treatment for caregivers who are experiencing depression. Given the key role primary care plays in delivering mental health care, ${ }^{38,} 39$ this information may be particularly useful for primary care practice. For example, asking about caregiving responsibilities may enable primary care providers to identify high-risk patients who will benefit from close follow-up to maximize the probability that they persist with their depression treatment. Enhancing mental health referrals with active follow-up may also encourage uptake of recommended treatments, and may be particularly important in a rural population.

Oncology and specialty teams may also have a role in supporting co-survivors' mental health, by integrating cosurvivors into family-centered cancer care. Several models for such integration are beginning to emerge, including multidisciplinary hospital-based caregiver clinics, ${ }^{40}$ caregiver supports through palliative and hospice care, ${ }^{41}$ and the Family Caregiver Program at the Department of Veterans Affairs. ${ }^{42}$ Most organizations, however, do not yet have a formal, standardized mechanism for integrating co-survivors into cancer care. ${ }^{43}$ Early involvement of palliative care-with an additional emphasis on evaluating caregiver depression and stress - may also facilitate optimal mental health care for co-survivors.

Finally, enhanced patient education may improve depression treatment outcomes for co-survivors. Addressing misconceptions about the efficacy and purpose of depression treatment - in general and for caregivers specifically - may reduce feelings of futility and improve depression treatment plan adherence. Such education may be particularly critical for rural co-survivors, for whom stigma and negative beliefs about depression and treatment may be particularly relevant. ${ }^{36}$ Furthermore, community- 
level approaches to decreasing cultural barriers such as stigma, or increasing access to mental health care may benefit all communities, and rural communities in particular.

This study should be interpreted in light of several limitations. Our definition of guideline-concordant depression treatment was guided by several assumptions and estimates made necessary by the dataset. Most panels of the MEPS do not include questions about caregiving; actual caregiving involvement (i.e., spouses' provision of emotional, function, or financial support) is therefore assumed rather than documented. We could not assess subjects' adherence to treatment or determine the appropriateness of guideline non-concordant treatment on an individual level. This study combined data across cancer types and we were unable to isolate cancer stage; carcinomas in situ were therefore included along with later stage cancers. There are some differences between the characteristics of the co-survivor and comparison groups. We opted to control for these possible confounders in the statistical analysis rather than through matching procedures. Finally, we were only able to examine spouses in this dataset; the findings may therefore not be generalizable to other caregiving populations or relationships. Nevertheless, this study makes use of a large, national dataset with household-level data, and provides compelling, generalizable evidence of differences in treatment for co-surviving spouses with depression, particularly in rural areas. The findings should therefore spur future research to determine these differences and the long-term impacts of such disparities with more precision.

In conclusion, this study found that spouses of cancer survivors were less likely to receive guideline-concordant treatment for depression than the general population of married adults. Those in rural areas were at particularly high risk of potential under-treatment. The findings highlight the need for enhanced follow-up among cancer co-survivors, to encourage uptake of and adherence to depression treatment. Innovative approaches to addressing barriers to mental health care, including family-centered cancer care and community-level approaches to depression education and stigma reduction, may also improve co-survivor outcomes, particularly in rural areas. Future research should examine and seek to mitigate clinical-, community-, and individual-level barriers to depression care for cancer co-survivors, with a focus on how the barriers and solutions may differ by rurality.

Acknowledgements: This study was funded in part by the American Cancer Society (IRG-15-213-51). Support was also received from the UW Carbone Cancer Center (NIH P3O CAO1452O). An earlier version of the manuscript was presented as a poster at the 38th Annual Meeting of the Society of Behavioral Medicine, in San Diego, CA, in 2017.

Corresponding Author: Kristin Litzelman, PhD; UW Carbone Cancer Center, Madison, WI, USA (e-mail: Litzelman@wisc.edu).

\section{Compliance with Ethical Standards:}

Conflict of Interest: The authors declare that they have no conflict of interest.

\section{REFERENCES}

1. Bluethmann SM, Mariotto AB, Rowland JH. Anticipating the "Silver Tsunami": prevalence trajectories and comorbidity burden among older cancer survivors in the United States. AACR; 2016.

2. National Coalition for Cancer Survivorship. Defining cancer survivorship. Cancer Policy Matters 2014.

3. Ganz PA. A teachable moment for oncologists: cancer survivors, 10 million strong and growing! J Clin Oncol 2005;23(24):5458-60. https:// doi.org/10.1200/JCO.2005.04.916

4. Deshields TL, Applebaum AJ. The time is now: assessing and addressing the needs of cancer caregivers. Cancer 2015;121(9):1344-6.

5. Hirst M. Carer distress: a prospective, population-based study. Soc Sci Med 2005;61(3):697-708. https://doi.org/10.1016/j.socscimed.2005. 01.001

6. Lowenstein A, Gilbar O. The perception of caregiving burden on the part of elderly cancer patients, spouses and adult children. Fam Syst Health. 2000;18(3):337.

7. Goren A, Gilloteau I, Lees M, DaCosta Dibonaventura M. Quantifying the burden of informal caregiving for patients with cancer in Europe. Support Care Cancer 2014;22(6):1637-46. https://doi.org/10.1007/ s00520-014-2122-6

8. Stenberg U, Ruland CM, Miaskowski C. Review of the literature on the effects of caring for a patient with cancer. Psychooncology 2010;19(10):1013-25. https://doi.org/10.1002/pon.1670

9. Litzelman K, Green PA, Yabroff KR. Cancer and quality of life in spousal dyads: spillover in couples with and without cancer-related health problems. Support Care Cancer 2016;24(2):763-71.

10. Litzelman $\mathbf{K}$, Yabroff $\mathbf{K R}$. How are spousal depressed mood, distress, and quality of life associated with risk of depressed mood in cancer survivors? Longitudinal findings from a national sample. Cancer Epidemiol Biomark Prev 2015;24(6):969-77. https://doi.org/10.1158/ 1055-9965.EPI-14-1420

11. Northouse LL, Katapodi MC, Schafenacker AM, Weiss D. The impact of caregiving on the psychological well-being of family caregivers and cancer patients. Semin Oncol Nurs 2012;28(4):236-45. https://doi.org/10. 1016/j.soncn.2012.09.006

12. DiMatteo MR, Lepper HS, Croghan TW. Depression is a risk factor for noncompliance with medical treatment: meta-analysis of the effects of anxiety and depression on patient adherence. Arch Intern Med 2000;160(14):2101-7.

13. Colleoni M, Mandala M, Peruzzotti G, Robertson C, Bredart A, Goldhirsch A. Depression and degree of acceptance of adjuvant cytotoxic drugs. Lancet 2000;356(9238):1326-7. https://doi.org/10.1016/S01406736(00)02821-X

14. Stanton AL, Petrie KJ, Partridge AH. Contributors to nonadherence and nonpersistence with endocrine therapy in breast cancer survivors recruited from an online research registry. Breast Cancer Res Treat 2014;145(2):525-34. https://doi.org/10.1007/s10549-014-2961-3

15. Pinquart M, Duberstein PR. Depression and cancer mortality: a metaanalysis. Psychol Med 2010;40(11):1797-810. https://doi.org/10.1017/ S0033291709992285

16. Satin J, Linden W, Phillips M. Depression as a predictor of disease progression and mortality in cancer patients: a meta-analysis. Cancer 2009;115(22):5349.

17. Mols F, Husson O, Roukema JA, van de Poll-Franse LV. Depressive symptoms are a risk factor for all-cause mortality: results from a prospective population-based study among 3,080 cancer survivors from the PROFILES registry. J Cancer Surviv: Res Pract 2013;7(3):484-92. https://doi.org/10.1007/s11764-013-0286-6

18. Cuijpers P, Sijbrandij M, Koole SL, Andersson G, Beekman AT, Reynolds CF. The efficacy of psychotherapy and pharmacotherapy in treating depressive and anxiety disorders: a meta-analysis of direct comparisons. World Psychiatry 2013;12(2):137-48.

19. Gonzalez HM, Vega WA, Williams DR, Tarraf W, West BT, Neighbors HW. Depression care in the United States: too little for too few. Arch Gen Psychiatry 2010;67(1):37-46. https://doi.org/10.1001/ archgenpsychiatry.2009.168

20. Seal K, Murray CD, Seddon L. The experience of being an informal "carer" for a person with cancer: a meta-synthesis of qualitative studies. Palliat Support Care. 2015;13(3):493-504. https://doi.org/10.1017/ S1478951513001132

21. Witt WP, Keller A, Gottlieb C, Litzelman K, Hampton J, Maguire J, et al. Access to adequate outpatient depression care for mothers in the USA: a nationally representative population-based study. J Behav Health 
Serv Res 2011;38(2):191-204. https://doi.org/10.1007/s11414-0099194-y

22. Bolin JN, Bellamy G. Rural healthy people 2020. College Station: The Texas A\&M Health Science Center, School of Rural Public Health, Southwest Rural Health Research Center. 2011.

23. Gelenberg AJ, Freeman MP, Markowitz JC, Rosenbaum JF, Thase ME, Trivedi MH, et al. Practice guideline for the treatment of patients with major depressive disorder third edition. Am J Psychiatr 2010; 167(10): 1 .

24. Kessler RC, Berglund P, Demler O, Jin R, Koretz D, Merikangas KR, et al. The epidemiology of major depressive disorder: results from the National Comorbidity Survey Replication (NCS-R). JAMA 2003;289(23):3095-105.

25. Harman JS, Edlund MJ, Fortney JC. Disparities in the adequacy of depression treatment in the United States. Psychiatr Serv 2004;55(12): 1379-85. https://doi.org/10.1176/appi.ps.55.12.1379

26. Keller AO, Gangnon R, Witt WP. The impact of patient-provider communication and language spoken on adequacy of depression treatment for U.S. women. Health Commun 2014;29(7):646-55. https://doi. org/10.1080/10410236.2013.795885

27. Cerner. Multim Lexicon Drug Database. https://www.cerner.com/solutions/drug-database. Accessed December 182017.

28. Andersen RM. Revisiting the behavioral model and access to medical care: does it matter? J Health Soc Behav 1995;36(1): 1-10.

29. Kroenke K, Spitzer RL, Williams JB. The Patient Health Questionnaire2: validity of a two-item depression screener. Med Care 2003;41(11):1284-92. https://doi.org/10.1097/01.MLR.0000093487. 78664.3C

30. Mosher CE, Champion VL, Hanna N, Jalal SI, Fakiris AJ, Birdas TJ, et al. Support service use and interest in support services among distressed family caregivers of lung cancer patients. Psycho-Oncology 2013;22(7):1549-56. https://doi.org/10.1002/pon.3168

31. Olfson M, Blanco C, Marcus SC. Treatment of adult depression in the United States. JAMA Intern Med 2016;176(10):1482-91. https://doi.org/ $10.1001 /$ jamainternmed.2016.5057

32. Mosher CE, Given BA, Ostroff JS. Barriers to mental health service use among distressed family caregivers of lung cancer patients. Eur J Cancer Care 2015;24(1):50-9. https://doi.org/10.1111/ecc. 12203
33. Fortney JC, Harman JS, Xu S, Dong F. The association between rural residence and the use, type, and quality of depression care. J Rural Health 2010;26(3):205-13. https://doi.org/10.1111/j.1748-0361.2010. 00290.x

34. U.S. Department of Health and Human Services, Health Resources and Services Administration. Designated Health Professional shortage Areas (HPSA) Statistics2012.

35. Bolin JN, Bellamy G. Rural healthy people 20202011

36. Brenes GA, Danhauer SC, Lyles MF, Hogan PE, Miller ME. Barriers to mental health treatment in rural older adults. Am J Geriatr Psychiatry 2015;23(11):1172-8. https://doi.org/10.1016/j.jagp.2015.06.002

37. Northouse LL, Katapodi MC, Song $\mathbf{L}$, Zhang $\mathbf{L}$, Mood DW. Interventions with family caregivers of cancer patients: meta-analysis of randomized trials. CA Cancer J Clin 2010;60(5):317-39

38. Regier DA, Goldberg ID, Taube CA. The de facto US mental health services system: a public health perspective. Arch Gen Psychiatry 1978;35(6):685-93.

39. World Health Organization, World Organization of National Colleges, Academic Associations of General Practitioners/Family Physicians. Integrating mental health into primary care: a global perspective: World Health Organization; 2008.

40. Applebaum AJ, Kulikowski JR, Breitbart W. Meaning-Centered Psychotherapy for Cancer Caregivers (MCP-C): rationale and overview. Palliat Support Care 2015;13(6):1631-41. https://doi.org/10.1017/ S1478951515000450

41. Oliver DP, Albright DL, Kruse RL, Wittenberg-Lyles E, Washington K, Demiris G. Caregiver evaluation of the ACTIVE intervention: "it was like we were sitting at the table with everyone". Am J Hosp Palliat Care 2014;31(4):444-53. https://doi.org/10.1177/1049909113490823

42. Wright P, Malcolm C, Hicken B, Rupper R. The VA caregiver support line: a gateway of support for caregivers of veterans. J Gerontol Soc Work 2015;58(4):386-98. https://doi.org/10.1080/01634372.2015.1019168

43. Kent EE, Rowland JH, Northouse L, Litzelman K, Chou WY, Shelburne N, et al. Caring for caregivers and patients: research and clinical priorities for informal cancer caregiving. Cancer 2016;122(13):1987-95. https://doi.org/10.1002/cncr.29939 